TRANSACTIONS OF THE

AMERICAN MATHEMATICAL SOCIETY

Volume 362, Number 3, March 2010, Pages 1169-1187

S 0002-9947(09)04542-5

Article electronically published on October 15, 2009

\title{
POWERS OF LARGE RANDOM UNITARY MATRICES AND TOEPLITZ DETERMINANTS
}

\author{
MAURICE DUITS AND KURT JOHANSSON
}

\begin{abstract}
We study the limiting behavior of $\operatorname{Tr} U^{k(n)}$, where $U$ is an $n \times n$ random unitary matrix and $k(n)$ is a natural number that may vary with $n$ in an arbitrary way. Our analysis is based on the connection with Toeplitz determinants. The central observation of this paper is a strong Szegö limit theorem for Toeplitz determinants associated to symbols depending on $n$ in a particular way. As a consequence of this result, we find that for each fixed $m \in$ $\mathbb{N}$, the random variables $\operatorname{Tr} U^{k_{j}(n)} / \sqrt{\min \left(k_{j}(n), n\right)}, j=1, \ldots, m$, converge to independent standard complex normals.
\end{abstract}

\section{InTRODUCTION AND STATEMENT OF RESULtS}

Random matrix theory. Let $U$ be a random unitary matrix with respect to the Haar measure on $U(n)$, where $U(n)$ is the group of unitary matrices of size $n \times n$. Denote the eigenvalues of $U$ by $\mathrm{e}^{\mathrm{i} \theta_{\mu}}$, for $\mu=1, \ldots, n$ with $\theta_{\mu} \in[-\pi, \pi)$. Throughout this paper we will consider the random variable $X_{n}$ defined by

$$
X_{n}(U)=\sum_{\mu=1}^{n} f_{n}\left(\mathrm{e}^{\mathrm{i} \theta_{\mu}}\right)
$$

where $f_{n}$ is a square integrable function on $\mathbb{T}=\{z \in \mathbb{C}:|z|=1\}$ with Fourier series

$$
f_{n}(z)=\sum_{|j|>0} \frac{\alpha_{j} z^{k_{j}(n)}}{\sqrt{\min \left(\left|k_{j}(n)\right|, n\right)}} .
$$

Here we assume that $\left\{\alpha_{j}\right\}_{j \in \mathbb{Z}}$ is a square summable sequence satisfying $\alpha_{-j}=\overline{\alpha_{j}}$, for each $n \in \mathbb{N}$ the sequence $\left\{k_{j}(n)\right\}_{j \in \mathbb{N}}$ consists of mutually distinct positive integers and $k_{-j}(n)=-k_{j}(n)$. Under these conditions $f_{n}$ is real-valued. Alternatively, we may write $X_{n}$ as

$$
X_{n}(U)=\sum_{|j|>0} \frac{\alpha_{j}}{\sqrt{\min \left(\left|k_{j}(n)\right|, n\right)}} \operatorname{Tr} U^{k_{j}(n)} .
$$

The main result we obtain is the following theorem.

Received by the editors July 11, 2006 and, in revised form, April 24, 2007.

2000 Mathematics Subject Classification. Primary 60B15; Secondary 47B35, 15A52, 60F05.

The first author is a research assistant of the Fund for Scientific Research-Flanders and was supported by the Marie Curie Training Network ENIGMA, European Science Foundation Program MISGAM, FWO-Flanders project G.0455.04, K.U. Leuven research grant OT/04/21 and Belgian Interuniversity Attraction Pole P06/02.

The second author was supported by the Göran Gustafsson Foundation (KVA).

(C)2009 American Mathematical Society 
Theorem 1.1. We have that

$$
\lim _{n \rightarrow \infty} \mathbb{E}\left[\mathrm{e}^{\mathrm{i} X_{n}}\right]=\mathrm{e}^{-\sum_{j=1}^{\infty}\left|\alpha_{j}\right|^{2}}
$$

Hence, for each fixed $m \in \mathbb{N}$, the random variables $\frac{1}{\sqrt{\min \left(k_{j}(n), n\right)}} \operatorname{Tr} U^{k_{j}(n)}$, for $j=1, \ldots, m$, converge to independent standard complex normals.

The latter result was obtained before in several special cases. When $k_{j}(n)$, $1 \leq j \leq m$, do not depend on $n$, this result is proved by Diaconis and Shahshahani [7] using moment identities. In this case it is in fact a direct consequence of the strong Szegö limit theorem for Toeplitz determinants and the Weyl integration formula. If we consider a single $k_{j}(n) \geq n$ this result is due to Rains; see [11. More details and an extensive list of references can be found in the survey article by Diaconis [5]. See also Diaconis and Evans [6].

There is a remarkable difference in normalization between the two cases $k_{j}(n) \leq$ $n$ and $k_{j}(n)>n$. For the single case $k_{j}(n)>n$, Rains proved that the eigenvalues of $U^{k_{j}(n)}$ behave like $n$ independently and uniformly distributed points on the unit circle. Therefore (1.1) follows from the classical central limit theorem. In particular, the sum of the eigenvalues is of order $\sqrt{n}$.

For $k_{j}(n) \leq n$, the term $\operatorname{Tr} U^{k_{j}(n)}$ is normalized by $\sqrt{k_{j}(n)}$. This normalization follows from the correlation between the eigenvalues of $U^{k_{j}(n)}$. Due to repulsion, the typical picture one finds for the eigenvalues is that of a small perturbation of $n$ equidistant points on the unit circle and we have a very effective cancellation. Note that the sum of $n$ equidistant points on the unit circle is zero.

Our result generalizes previous results by allowing arbitrary powers depending on $n$ and thus combines the result from Szegö's theorem with that of Rains.

An interesting generalization of the problem we consider would be to allow the coefficients $\alpha_{j}$ to depend on $n$. In this case it seems difficult to formulate a general theorem. See section 5 for a remark.

Strong Szegö limit for $n$-dependent symbols. The starting point of our analysis is the connection with Toeplitz determinants. If $a \in \mathbb{L}_{1}(\mathbb{T})$, let $T_{n}(a)$ be the $n \times n$ matrix given by $\left(T_{n}(a)\right)_{j k}=a_{j-k}$, where the $a_{k}$ are the Fourier coefficients of $a$. The Heine-Szegö identity states that

$$
\mathbb{E}\left[\mathrm{e}^{\mathrm{i} X_{n}}\right]=\operatorname{det} T_{n}\left(\mathrm{e}^{\mathrm{i} f_{n}}\right) ;
$$

see [5]. Using this identity we see that in case $k_{j}(n), 1 \leq j \leq m$, do not depend on $n$, Theorem 1.1 is nothing else than the strong Szegö limit for Toeplitz determinants. In order to prove Theorem 1.1 in the general case, we will prove a strong Szegö limit for $n$-dependent symbols of the type (1.2).

Note that $f_{n}$ as defined in (1.2) is a real-valued function. The strong Szegö limit that we prove holds for complex-valued functions as well, but with a stronger condition on the coefficients $\alpha_{j}$. For the sake of completeness we will prove the general complex-valued case.

Let $\left\{\alpha_{j}\right\}_{j \in \mathbb{Z}}$ be any sequence of complex numbers satisfying $\sum_{j}\left|\alpha_{j}\right|<\infty$. For each $n \in \mathbb{N}$ let $\left\{k_{j}(n)\right\}_{j \in \mathbb{N}}$ again be a sequence of mutually distinct positive integers 
and set $k_{-j}(n)=-k_{j}(n)$. Define $g_{n}: \mathbb{T} \rightarrow \mathbb{C}$ by

$$
g_{n}(z)=\sum_{|j|>0} \frac{\alpha_{j} z^{k_{j}(n)}}{\sqrt{\min \left(\left|k_{j}(n)\right|, n\right)}},
$$

for all $z \in \mathbb{T}$ and $n \in \mathbb{N}$. Our main result is the following.

Theorem 1.2. If $\sum_{j}\left|\alpha_{j}\right|<\infty$, then

$$
\lim _{n \rightarrow \infty} \operatorname{det} T_{n}\left(\mathrm{e}^{g_{n}}\right)=\exp \sum_{j=1}^{\infty} \alpha_{j} \alpha_{-j} .
$$

This is the analogue of the strong Szegö theorem for Toeplitz determinants, but now for symbols that vary with $n$ in a particular way.

Now Theorem 1.1 follows from (1.5) and Theorem 1.2 with $g_{n}=\mathrm{i} f_{n}$, but under the extra condition $\sum_{j}\left|\alpha_{j}\right|<\infty$. This condition can however be eliminated by a standard approximation argument, which is described in Section 4 . However, we want to emphasize that this argument depends on the fact that $f_{n}$ is real-valued.

Overview of the proof. We will omit the dependence on $n$ in the notation and simply write $g$ and $k_{j}$. Split $g$ into

$$
g(z)=g^{(1)}(z)+g^{(2)}(z)=\sum_{0<\left|k_{j}\right| \leq n} \frac{\alpha_{j} z^{k_{j}}}{\sqrt{\left|k_{j}\right|}}+\sum_{\left|k_{j}\right|>n} \frac{\alpha_{j} z^{k_{j}}}{\sqrt{n}} .
$$

Let $a$ and $b$ be defined by

$$
a=\mathrm{e}^{g^{(1)}} \quad \text { and } \quad b=\mathrm{e}^{g^{(2)}} .
$$

Define

$$
C^{(1)}=\sum_{0<k_{j} \leq n} \alpha_{j} \alpha_{-j}, \quad C^{(2)}=\sum_{k_{j}>n} \alpha_{j} \alpha_{-j}, \quad C=\sum_{j=1}^{\infty} \alpha_{j} \alpha_{-j} .
$$

Note that $C^{(1)}$ and $C^{(2)}$ depend on $n$, whereas $C$ does not.

The terms $a$ and $b$ are very different in behavior. As a consequence, we analyze them separately. We therefore divide the proof of Theorem 1.2 into two parts. The first part consists of proving that

$$
\lim _{n \rightarrow \infty} \mathrm{e}^{-C^{(1)}} \operatorname{det} T_{n}(a)=1 .
$$

To this end we need the Fredholm determinant identity for Toeplitz determinants, which was found by Case and Geronimo [8] and independently by Borodin and Okounkov 3 .

The second part consists of proving that

$$
\lim _{n \rightarrow \infty} \frac{\mathrm{e}^{-C^{(2)}} \operatorname{det} T_{n}(a b)}{\operatorname{det} T_{n}(a)}=1 .
$$


Indeed if we can prove that (1.11) and (1.12) hold, then a simple multiplication of the two gives

$$
\lim _{n \rightarrow \infty} \mathrm{e}^{-C^{(1)}-C^{(2)}} \operatorname{det} T_{n}(a b)=\lim _{n \rightarrow \infty} \mathrm{e}^{-C} \operatorname{det} T_{n}(a b)=1 .
$$

Now, since $C$ does not depend on $n$ we can multiply both sides with $\mathrm{e}^{C}$, which proves Theorem 1.2 ,

For reasons of clarity we will prepare the proof of (1.12) and first prove

$$
\lim _{n \rightarrow \infty} \mathrm{e}^{-C^{(2)}} \operatorname{det} T_{n}(b)=1 .
$$

The proof of this result follows by a fairly direct computation. The results of this computation can be used for proving (1.12). Hence, in the remaining proof of (1.12) we can restrict ourselves to only those parts that come in by interaction of $g^{(1)}$ and $g^{(2)}$. In our opinion, it helps to get a better understanding of the problem. Moreover, combining (1.11), (1.12) and (1.14) we immediately find the following result.

Proposition 1.3. We have that

$$
\lim _{n \rightarrow \infty} \frac{\operatorname{det} T_{n}(a b)}{\operatorname{det} T_{n}(a) \operatorname{det} T_{n}(b)}=1 .
$$

This is a so-called separation theorem. Such results have often been investigated before; see for example 2, 14. However, all the results known thus far use the fact that $H(a) H(\tilde{b})$ is of trace class. This is not necessarily true in our case, which makes Theorem 1.3 an interesting result in its own right.

\section{Preliminaries}

To fix notation, we recall some definitions of certain operators and Banach algebras we need later. For a more detailed discussion we refer to [4].

For $c \in \mathbb{L}_{\infty}(\mathbb{T})$, define infinite matrices $T(c)$ and $H(c)$ by

$$
T(c)=\left(c_{j-l}\right)_{j, l=1}^{\infty} \quad \text { and } \quad H(c)=\left(c_{j+l-1}\right)_{j, l=1}^{\infty},
$$

where $c_{k}$ are the Fourier coefficients of $c$. These matrices induce bounded operators on $\ell_{2}(\mathbb{N})$. Moreover, $\|T(c)\|_{\infty}=\|c\|_{\mathbb{L}_{\infty}}$ and $\|H(c)\|_{\infty} \leq\|c\|_{\infty}$.

Denote with $P_{n}$ the projection operator on $\ell_{2}$ that projects on the subspace of all $x \in \ell_{2}(\mathbb{N})$ for which $x_{k}=0$ for all $k>n$. Define $Q_{n}=I-P_{n}$. Let $W_{n}: \ell_{2}(\mathbb{N}) \rightarrow \ell_{2}(\mathbb{N})$ be the operator defined by

$$
\left(W_{n} x\right)_{k}=\left\{\begin{array}{cc}
x_{n-k+1}, & 1 \leq k \leq n \\
0, & k>n
\end{array}\right.
$$

for all $x \in \ell_{2}(\mathbb{N})$. If $c \in \mathbb{L}_{\infty}$, then

$$
W_{n} T_{n}(c) W_{n}=T_{n}(\tilde{c}),
$$

where $\tilde{c}(z)=c(1 / z)$.

Next we recall the definition of certain Banach algebras which will appear frequently in the sequel. 
The space $B_{2}^{1 / 2}$ consists of all $f \in \mathbb{L}_{2}(\mathbb{T})$ for which $\sum_{k}|k|\left|f_{k}\right|^{2}<\infty$, equipped with the norm defined by

$$
\|f\|_{B_{2}^{1 / 2}}^{2}=\sum_{k}(1+|k|)\left|f_{k}\right|^{2} .
$$

Again, the $f_{k}$ denote the Fourier coefficients of $f$. The space $B_{2}^{1 / 2}$ is a Sobolev space.

The Wiener algebra consists of all $f \in \mathbb{L}_{\infty}$, for which $\sum_{k}\left|f_{k}\right|<\infty$ and has norm

$$
\|f\|_{W}=\sum_{k}\left|f_{k}\right|,
$$

for all $f \in W$. It is well known that this is a Banach algebra.

The space $W \cap B_{2}^{1 / 2}$ equipped with the norm

$$
\|f\|_{W \cap B_{2}^{1 / 2}}=\|f\|_{W}+\|f\|_{B_{2}^{1 / 2}}
$$

for $f \in W \cap B_{2}^{1 / 2}$ is also a Banach algebra.

Note that due to the assumption $\sum\left|\alpha_{j}\right|<\infty$ we have that $g^{(1)} \in W \cap B_{2}^{1 / 2}$ and $g^{(2)} \in W$. In particular this shows that $a$ and $b$ in (1.9) are well-defined. Moreover, $a \in W \cap B_{2}^{1 / 2}, b \in W$ and we have the following inequalities:

$$
\begin{aligned}
\|a\|_{B_{2}^{1 / 2}} & \leq \mathrm{e}^{\left\|g^{(1)}\right\|_{W \cap B_{2}^{1 / 2}}}<\exp \left(A_{1}+\sqrt{2} A_{2}\right) \\
\|b\|_{W} & \leq \mathrm{e}^{\left\|g^{(2)}\right\|_{W}} \leq \mathrm{e}^{\sum\left|\alpha_{j}\right| / \sqrt{n}} .
\end{aligned}
$$

Hence, $\|a\|_{B_{2}^{1 / 2}}$ and $\|b\|_{W}$ are uniformly bounded in $n$. For convenience we define

$$
A_{1}=\sum\left|\alpha_{j}\right| \quad \text { and } \quad A_{2}=\left(\sum\left|\alpha_{j}\right|^{2}\right)^{1 / 2}
$$

These constants will appear frequently in upcoming inequalities.

Besides the operator norm $\|\cdot\|_{\infty}$ we will also use the trace norm, denoted by $\|\cdot\|_{1}$, and the Hilbert-Schmidt norm, denoted by $\|\cdot\|_{2}$. Note that if $c \in W \cap B_{2}^{1 / 2}$, then $H(c)$ is a Hilbert-Schmidt operator and

$$
\|H(c)\|_{2}^{2}=\sum_{j, l=1}^{\infty}\left|c_{j+l-1}\right|^{2}=\sum_{j=1}^{\infty} j\left|c_{j}\right|^{2} \leq\|c\|_{B_{2}^{1 / 2}}^{2}
$$

This will be used frequently in the sequel.

\section{Proof of Theorem 1.2}

3.1. Proof of (1.11). First, we will prove (1.11). To this end we will use a celebrated Fredholm identity for Toeplitz determinants. Let $g_{+}^{(1)}$ be the projection of $g^{(1)}$ onto the subspace of all $f \in W \cap B_{2}^{1 / 2}$ for which $f_{k}=0$ for all $k<0$. Moreover, define $g_{-}^{(1)}=g^{(1)}-g_{+}^{(1)}, a_{+}=\mathrm{e}^{g_{+}^{(1)}}$ and $a_{-}=\mathrm{e}^{g_{-}^{(1)}}$. Finally, define $\phi=a_{+}^{-1} a_{-}$and $\psi=\widetilde{a_{+}} \widetilde{a_{-}^{-1}}$.

The Borodin-Okounkov-Geronimo-Case identity now states that

$$
\operatorname{det} T_{n}(a)=\mathrm{e}^{C^{(1)}} \operatorname{det}\left(I-Q_{n} H(\phi) H(\psi) Q_{n}\right),
$$


for all $n \in \mathbb{N}$. Note that since $W \cap B_{2}^{1 / 2}$ is a Banach algebra, we find that $\phi, \psi \in$ $W \cap B_{2}^{1 / 2}$ and hence $Q_{n} H(\phi) H(\psi) Q_{n}$ is a trace-class operator. The determinant on the right-hand side is a Fredholm determinant. Note that we use the formulation by Basor and Widom, see [1, which is slightly different from the one by Borodin and Okounkov in 3 .

So we need to prove that the Fredholm determinant converges to 1 to obtain (1.11).

Lemma 3.1. We have that

$\left|\operatorname{det}\left(I-Q_{n} H(\phi) H(\psi) Q_{n}\right)-1\right| \leq \exp \left(\left(\sum_{k=1}^{\infty} k\left|\phi_{k+n}\right|^{2}\right)^{1 / 2}\left(\sum_{k=1}^{\infty} k\left|\psi_{k+n}\right|^{2}\right)^{1 / 2}\right)-1$,

for all $n \in \mathbb{N}$.

Proof. A standard inequality for Fredholm determinants gives

$$
\left|\operatorname{det}\left(I-Q_{n} H(\phi) H(\psi) Q_{n}\right)-1\right| \leq \mathrm{e}^{\left\|Q_{n} H(\phi) H(\psi) Q_{n}\right\|_{1}}-1 .
$$

The trace norm can be estimated by

$$
\left\|Q_{n} H(\phi) H(\psi) Q_{n}\right\|_{1} \leq\left\|Q_{n} H(\phi)\right\|_{2}\left\|H(\psi) Q_{n}\right\|_{2} .
$$

A straightforward calculation shows that

$$
\left\|Q_{n} H(\phi)\right\|_{2}^{2}=\sum_{k=1}^{\infty} k\left|\phi_{k+n}\right|^{2} \quad \text { and } \quad\left\|H(\psi) Q_{n}\right\|_{2}^{2}=\sum_{k=1}^{\infty} k\left|\psi_{k+n}\right|^{2},
$$

which proves the statement.

Hence we need to show that

$$
\lim _{n \rightarrow \infty} \sum_{k=1}^{\infty} k\left|\phi_{k+n}\right|^{2}=0 \quad \text { and } \quad \lim _{n \rightarrow \infty} \sum_{k=1}^{\infty} k\left|\psi_{k+n}\right|^{2}=0 .
$$

Note that if $\phi$ and $\psi$ did not depend on $n$ (as in the classical case), then this trivially holds. But since they depend on $n$ there is still some work to be done.

Lemma 3.2. Let $N \in \mathbb{N}$ and $t$ be defined by the Fourier series $t(z)=\sum_{0<|j| \leq N} \frac{t_{j} z^{j}}{\sqrt{|j|}}$. Define $F_{t}$ associated to $t$ by $F_{t}(z)=\sum_{0<|j| \leq N}\left|t_{j}\right| z^{j}$. Then

$$
\left|\left(\mathrm{e}^{t}\right)_{k+N}\right|<\frac{1}{\sqrt{k(N+k)}}\left(F_{t}\left(\mathrm{e}^{F_{t}}-1\right)\right)_{N+k}
$$

for all $k \in \mathbb{N}$.

Proof. First consider powers $t^{l}$ for $l \geq 2$. Then

$$
\left(t^{l}\right)_{k+N}=\sum_{j_{1}+j_{2}+\cdots+j_{l}=k+N} \frac{t_{j_{1}} \cdots t_{j_{l}}}{\sqrt{\left|j_{1} \cdots j_{l}\right|}}
$$

Since $j_{1}+j_{2}+\cdots+j_{l}=k+N$, there should be at least one $j_{s}$, with $j_{s} \geq(N+k) / l$. But $j_{s} \leq N$ and hence $j_{1}+j_{2}+\cdots+j_{l}-j_{s} \geq k$. Hence there exists a $j_{r} \neq j_{s}$ such that $j_{r} \geq k /(l-1)>k / l$. 
Therefore

$$
\left|\left(t^{l}\right)_{k+N}\right|<\frac{l}{\sqrt{k(k+N)}} \sum_{j_{1}+j_{2}+\cdots+j_{l}=k+N}\left|t_{j_{1}} \cdots t_{j_{l}}\right|=\frac{l}{\sqrt{k(k+N)}}\left(F_{t}^{l}\right)_{k+N} .
$$

Hence,

$$
\begin{aligned}
\left|\left(\mathrm{e}^{t}\right)_{k+N}\right| & \leq \sum_{l=2}^{\infty} \frac{\left|\left(t^{l}\right)_{k+N}\right|}{l !} \\
& <\sum_{l=2}^{\infty} \frac{\left(F_{t}^{l}\right)_{k+N}}{(l-1) ! \sqrt{k(k+N)}}=\frac{1}{\sqrt{k(k+N)}}\left(F_{t}\left(\mathrm{e}^{F_{t}}-1\right)\right)_{k+N} .
\end{aligned}
$$

This proves the statement.

Now we immediately find the following corollary.

Corollary 3.3. With $A_{1}$ as in (2.8) we have that

$$
\sum_{k=1}^{\infty} k\left|\phi_{k+n}\right|^{2}<\frac{A_{1}\left(\mathrm{e}^{A_{1}}-1\right)}{n}
$$

for all $n$. The same estimate holds for $\psi$.

Proof. Applying Lemma 3.2 with $t=\phi$ and $N=n$, we find

$$
\sum_{k=1}^{\infty} k\left|\phi_{k+n}\right|^{2}<\frac{\left\|F_{\phi}\left(\mathrm{e}^{F_{\phi}}-1\right)\right\|_{\mathbb{L}_{2}}^{2}}{n} .
$$

The statement now follows from the fact that $\|\cdot\|_{\mathbb{L}_{2}} \leq\|\cdot\|_{W}$, the fact that $W$ is a Banach algebra and $\left\|F_{\phi}\right\|_{W} \leq A_{1}$.

Now (1.11) follows by combining Corollary 3.3, Lemma 3.1 and (3.1).

3.2. Proof of (1.14). Next we analyze $\operatorname{det} T_{n}(b)$. In this case the identity (3.1) breaks down at two places. First, the factor in front of the Fredholm determinant is infinite, since $b$ is not necessarily contained in $W \cap B_{2}^{1 / 2}$. Second, the operator in the Fredholm determinant is no longer of trace class and the determinant is therefore not well-defined. However, there is no need for such a strong result as (3.1), since a direct analysis on $\operatorname{det} T_{n}(b)$ will suffice.

We will use the notion of regularized determinants. For a trace-class operator $A$ the regularized determinant is defined by

$$
\operatorname{det}_{2}(I+A)=\mathrm{e}^{-\operatorname{Tr} A} \operatorname{det}(I+A) .
$$

One can prove that $A \mapsto \operatorname{det}_{2}(I+A)$ is a continuous function defined on a dense subspace (namely the space of all trace-class operators) of the space of HilbertSchmidt operators. Therefore it can be extended and defined for all Hilbert-Schmidt operators. Moreover, we have that

$$
\left|\operatorname{det}_{2}(I+A)-1\right| \leq\|A\|_{2} \exp \left(\frac{1}{2}\left(\|A\|_{2}+1\right)^{2}\right),
$$

for all Hilbert-Schmidt operators. 
We will use the regularized determinant only for matrices, but (3.7) plays a crucial role. Write

$$
\operatorname{det} T_{n}(b)=\operatorname{det}\left(I+T_{n}(b-1)\right)=\mathrm{e}^{\operatorname{Tr} T_{n}(b-1)} \operatorname{det}_{2}\left(I+T_{n}(b-1)\right) .
$$

The proof of (1.14) falls into two parts. First we will show that the Hilbert-Schmidt norm of $T_{n}(b-1)$ tends to 0 as $n \rightarrow \infty$; hence the regularized determinant tends to 1 . Second, we show that $\operatorname{Tr} T_{n}(b-1)-C^{(2)}$ tends to 0 as $n \rightarrow \infty$. Then (1.14) follows by (3.7) and (3.8).

We start with the trace of $T_{n}(b-1)$. We define $g_{+}^{(2)}$ as $\sum_{\left|k_{j}\right|>n} \frac{\alpha_{j} z^{k_{j}}}{\sqrt{n}}$ and $g_{-}^{(2)}=g^{(2)}-g_{+}^{(2)}$. Moreover, we let $b_{ \pm}=\mathrm{e}^{g_{ \pm}^{(2)}}$.

Lemma 3.4. With $A_{1}$ as in (2.8) we have that

$$
\left|\operatorname{Tr} T_{n}(b-1)-C^{(2)}\right| \leq n\left(\mathrm{e}^{A_{1} / \sqrt{n}}-1\right)^{2}-A_{1}^{2},
$$

for all $n \in \mathbb{N}$.

Proof. First note that $\operatorname{Tr} T_{n}(b-1)=n\left(b_{0}-1\right)$. Now

$$
\begin{aligned}
b_{0}-1 & =\sum_{j \geq 0}\left(\mathrm{e}^{g_{+}^{(2)}}\right)_{j}\left(\mathrm{e}^{g_{-}^{(2)}}\right)_{-j}-1=\sum_{j \geq 0}\left(\mathrm{e}^{g_{+}^{(2)}}-1\right)_{j}\left(\mathrm{e}^{g_{-}^{(2)}}-1\right)_{-j} \\
& =\sum_{l=1}^{\infty} \sum_{m=1}^{\infty} \sum_{j \geq 0} \frac{\left(g_{+}^{(2)^{l}}\right)_{j}\left(g_{-}^{(2)^{m}}\right)_{-j}}{l ! m !} .
\end{aligned}
$$

Since $\sum_{j>n} \alpha_{j} \alpha_{-j}=n \sum_{j \geq 0}\left(g_{+}^{(2)}\right)_{j}\left(g_{-}^{(2)}\right)_{-j}$, we find

$$
\begin{aligned}
\frac{1}{n} \mid \operatorname{Tr} T_{n}(b-1) & -\sum_{j>n} \alpha_{j} \alpha_{-j}|=| b_{0}-1-\sum_{j \geq 0}\left(g_{+}^{(2)}\right)_{j}\left(g_{-}^{(2)}\right)_{-j} \mid \\
& =\left|\sum_{l=1}^{\infty} \sum_{m=1}^{\infty} \sum_{j \geq 0} \frac{\left(g_{+}^{(2)^{l}}\right)_{j}\left(g_{-}^{(2)^{m}}\right)_{-j}}{l ! m !}-\sum_{j \geq 0}\left(g_{+}^{(2)}\right)_{j}\left(g_{-}^{(2)}\right)_{-j}\right| .
\end{aligned}
$$

Now apply the Cauchy-Schwarz inequality to obtain

$$
\begin{aligned}
\frac{1}{n} \mid \operatorname{Tr} T_{n}(b-1) & -\sum_{j>n} \alpha_{j} \alpha_{-j} \mid \leq \sum_{l=1}^{\infty} \sum_{m=1}^{\infty} \frac{\left\|g_{+}^{(2)^{l}}\right\|_{\mathbb{L}_{2}}\left\|g_{-}^{(2)^{m}}\right\|_{\mathbb{L}_{2}}}{l ! m !}-\left\|g_{+}^{(2)}\right\|_{\mathbb{L}_{2}}\left\|g_{-}^{(2)}\right\|_{\mathbb{L}_{2}} \\
& \leq \sum_{l=1}^{\infty} \sum_{m=1}^{\infty} \frac{\left\|g_{+}^{(2)^{l}}\right\|_{W}\left\|g_{-}^{(2)^{m}}\right\|_{W}}{l ! m !}-\left\|g_{+}^{(2)}\right\|_{W}\left\|g_{-}^{(2)}\right\|_{W} \\
& \leq \sum_{l=1}^{\infty} \sum_{m=1}^{\infty} \frac{\left\|g_{+}^{(2)}\right\|_{W}^{l}\left\|g_{-}^{(2)}\right\|_{W}^{m}}{l ! m !}-\left\|g_{+}^{(2)}\right\|_{W}\left\|g_{-}^{(2)}\right\|_{W} .
\end{aligned}
$$

Now $\left\|g_{ \pm}^{(2)}\right\|_{W} \leq A_{1} / \sqrt{n}$ proves the statement.

Next we proceed with the Hilbert-Schmidt norm of $T_{n}(b-1)$. 
Lemma 3.5. With $A_{1}$ as in (2.8), we have that

$$
\left\|T_{n}(b-1)\right\|_{2} \leq \sqrt{n}\left(\mathrm{e}^{A_{1} / \sqrt{n}}-1\right)^{2},
$$

for all $n \in \mathbb{N}$.

Proof. Since $\left(b_{ \pm}-1\right)_{j}=0$ for $j=-n+1, \ldots, n-1$ we find

$$
\begin{aligned}
\left\|T_{n}(b-1)\right\|_{2}^{2} & \leq n \sum_{j=-n+1}^{n-1}\left|(b-1)_{j}\right|^{2}=n \sum_{j=-n+1}^{n-1}\left|\left(\left(b_{+}-1\right)\left(b_{-}-1\right)\right)_{j}\right|^{2} \\
& \leq n\left\|\left(b_{+}-1\right)\left(b_{-}-1\right)\right\|_{\mathbb{L}_{2}}^{2} \leq n\left\|\left(b_{+}-1\right)\left(b_{-}-1\right)\right\|_{W}^{2} \\
& \leq n\left\|b_{+}-1\right\|_{W}^{2}\left\|b_{-}-1\right\|_{W}^{2} \leq n\left(\mathrm{e}^{\left\|g_{+}^{(2)}\right\|_{W}}-1\right)^{2}\left(\mathrm{e}^{\left\|g_{-}^{(2)}\right\|_{W}}-1\right)^{2} .
\end{aligned}
$$

By $\left\|g_{ \pm}^{2}\right\|_{W} \leq A_{1} / \sqrt{n}$ we obtain the statement.

By Lemma 3.5 and (3.7) we obtain

$$
\lim _{n \rightarrow \infty} \operatorname{det}_{2}\left(I+T_{n}(b-1)\right)=1 .
$$

By substituting this into (3.8) and using Lemma 3.4 we obtain (1.14).

3.3. Proof of (1.12). Since we proved the result for the cases (1.11) and (1.14) in a completely different way, a natural way to deal with the general case is to split the two cases. To this end we use a factorization theorem due to Widom,

$$
T_{n}(a b)=T_{n}(a) T_{n}(b)+P_{n} H(a) H(\widetilde{b}) P_{n}+W_{n} H(\widetilde{a}) H(b) W_{n},
$$

and the operator $B_{n}$ defined by

$$
B_{n}=T_{n}\left(a^{-1}\right)-P_{n} H\left(a_{+}^{-1}\right) H\left(\widetilde{a_{-}^{-1}}\right) P_{n}-W_{n} H\left(\widetilde{a_{-}^{-1}}\right) H\left(a_{+}^{-1}\right) W_{n} .
$$

The operator $B_{n}$ is a good approximation of the inverse of $T_{n}(a)$. In the case that $a$ does not depend on $n$, this observation is due to Widom. Moreover, the operator can be used to prove the strong Szegö limit; see [4, 13]. We will prove that it is also a good approximation in our case. One can show, see [4, 13], that

$$
B_{n} T_{n}(a)=I+P_{n} H\left(a_{+}^{-1}\right) H\left(\widetilde{a_{-}^{-1}}\right) Q_{n} T(a) P_{n}+W_{n} H\left(\widetilde{a_{-}^{-1}}\right) H\left(a_{+}^{-1}\right) Q_{n} T(\tilde{a}) W_{n}
$$

for all $n \in \mathbb{N}$. Even in our case where $a$ depends on $n$, the operators on the right-hand side are small in the trace norm.

Lemma 3.6. We have

$$
\left\|B_{n} T_{n}(a)-I\right\|_{1}=\mathcal{O}\left(n^{-\frac{1}{2}}\right)
$$

for $n \rightarrow \infty$.

Proof. First note that

$$
\left\|P_{n} H\left(a_{+}^{-1}\right) H\left(\widetilde{a_{-}^{-1}}\right) Q_{n} T(a) P_{n}\right\|_{1} \leq\left\|P_{n} H\left(a_{+}^{-1}\right)\right\|_{2}\left\|H\left(\widetilde{a_{-}^{-1}}\right) Q_{n}\right\|_{2}\left\|T(a) P_{n}\right\|_{\infty} .
$$

Now

$$
\left\|P_{n} H\left(a_{+}^{-1}\right)\right\|_{2} \leq\left\|H\left(a_{+}^{-1}\right)\right\|_{2} \leq\left\|a_{+}^{-1}\right\|_{B_{2}^{1 / 2}}<\exp \left(\sqrt{2} A_{2}+A_{1}\right),
$$

and

$$
\left\|T(a) P_{n}\right\|_{\infty} \leq\|a\|_{\infty} \leq\|a\|_{W} \leq \exp \left(A_{1}\right)
$$


and finally

$$
\left.\left\|H\left(\widetilde{a_{-}^{-1}}\right) Q_{n}\right\|_{2}^{2}=\sum_{k=1}^{\infty} k \mid \widetilde{a_{-}^{-1}}\right)\left._{k+n}\right|^{2} .
$$

By Lemma 3.2 and the same arguments as in Corollary 3.3, the latter is $\mathcal{O}\left(n^{-1}\right)$, as $n \rightarrow \infty$. This proves the statement.

Therefore the following corollary is immediate.

Corollary 3.7. We have that

$$
\lim _{n \rightarrow \infty} \operatorname{det} B_{n} T_{n}(a)=1 .
$$

In view of this corollary, it is enough to show that

$$
\lim _{n \rightarrow \infty} \exp \left(-C^{(2)}\right) \operatorname{det} B_{n} T_{n}(a b)=1
$$

to prove (1.12). This will cover the rest of this section.

We will again use the regularized determinant. Write

$$
\operatorname{det} B_{n} T_{n}(a b)=\mathrm{e}^{\operatorname{Tr}\left(B_{n} T_{n}(a b)-I\right)} \operatorname{det}_{2} B_{n} T_{n}(a b) .
$$

In view of (3.8) and (3.7), to prove (3.17) it is enough to (1) prove that $B_{n} T_{n}(a b)-I$ converges to zero in the Hilbert-Schmidt norm and (2) calculate its trace.

If we introduce the notation

$$
E_{n}=-P_{n} H\left(a_{+}^{-1}\right) H\left(\widetilde{a_{-}^{-1}}\right) P_{n}-W_{n} H\left(\widetilde{a_{-}^{-1}}\right) H\left(a_{+}^{-1}\right) W_{n}
$$

and

$$
F_{n}=P_{n} H(a) H(\widetilde{b}) P_{n}+W_{n} H(\widetilde{a}) H(b) W_{n},
$$

and multiply (3.12) from the left with $B_{n}$ we find by (3.13),

$$
B_{n} T_{n}(a b)=B_{n} T_{n}(a) T_{n}(b)+T_{n}\left(a^{-1}\right) F_{n}+E_{n} F_{n} .
$$

We will analyze the three terms on the right-hand side separately. In the following lemma, we state results about the Hilbert-Schmidt norms and the trace of each of these three terms, except for the trace of $T_{n}\left(a^{-1}\right) F_{n}$. All the statements follow from earlier results. However, $\operatorname{Tr} T_{n}\left(a^{-1}\right) F_{n}$ is more subtle and needs some extra attention.

Lemma 3.8. We have that

(1) $\left\|B_{n} T_{n}(a) T_{n}(b)-I\right\|_{2} \rightarrow 0$

(2) $\left|\operatorname{Tr}\left(B_{n} T_{n}(a) T_{n}(b)-I\right)-C^{(2)}\right| \rightarrow 0$,

(3) $\left\|E_{n} F_{n}\right\|_{1} \rightarrow 0$

(4) $\left\|T_{n}\left(a^{-1}\right) F_{n}\right\|_{2} \rightarrow 0$

for $n \rightarrow \infty$.

Proof. (1) We estimate the Hilbert-Schmidt norm by

$$
\begin{aligned}
\left\|B_{n} T_{n}(a) T_{n}(b)-I\right\|_{2} & \leq\left\|\left(B_{n} T_{n}(a)-I\right) T_{n}(b)\right\|_{2}+\left\|T_{n}(b-1)\right\|_{2} \\
& \leq\left\|B_{n} T_{n}(a)-I\right\|_{2}\left\|T_{n}(b)\right\|_{\infty}+\left\|T_{n}(b-1)\right\|_{2} .
\end{aligned}
$$

Note that $\left\|T_{n}(b)\right\|_{\infty} \leq\|b\|_{\mathbb{L}_{\infty}} \leq\|b\|_{W}$. The statement now follows from Lemma 3.5. Lemma 3.6 and (2.7). 
(2) Note that

$$
\begin{aligned}
& \left|\operatorname{Tr}\left(B_{n} T_{n}(a) T_{n}(b)-I\right)-C^{(2)}\right| \\
& \quad \leq\left|\operatorname{Tr}\left(\left(B_{n} T_{n}(a)-I\right) T_{n}(b)\right)\right|+\left|\operatorname{Tr} T_{n}(b-1)-C^{(2)}\right| \\
& \quad \leq\left\|\left(B_{n} T_{n}(a)-I\right)\right\|_{1}\left\|T_{n}(b)\right\|_{\infty}+\left|\operatorname{Tr} T_{n}(b-1)-C^{(2)}\right| .
\end{aligned}
$$

The statement now follows from Lemma 3.4 and Lemma 3.6

(3) First note that $\left\|E_{n} F_{n}\right\|_{1} \leq\left\|E_{n}\right\|_{2}\left\|F_{n}\right\|_{2}$. Now

$$
\begin{aligned}
\left\|F_{n}\right\|_{2} & \leq\left\|P_{n} H(a)\right\|_{2}\left\|P_{n} H(\tilde{b})\right\|_{\infty}+\left\|W_{n} H(\tilde{a})\right\|_{2}\left\|H(b) W_{n}\right\|_{\infty} \\
& \leq\|a\|_{B_{2}^{1 / 2}}\|b-1\|_{\infty} \leq\|a\|_{B_{2}^{1 / 2}}\|b-1\|_{W} \\
& \leq\|a\|_{B_{2}^{1 / 2}}\left(\exp \left(A_{1} / \sqrt{n}\right)-1\right)
\end{aligned}
$$

with $A_{1}$ as in (2.8). By combining (3.22) with (2.6) we obtain $\left\|F_{n}\right\|_{2} \rightarrow 0$. By similar estimates one finds that $\left\|E_{n}\right\|_{2}$ is bounded in $n$.

(4) This follows from (3.22) and the following estimate: $\left\|T_{n}\left(a^{-1}\right) F_{n}\right\|_{2} \leq$ $\left\|T_{n}\left(a^{-1}\right)\right\|_{\infty}\left\|F_{n}\right\|_{2}$. Note that $\left\|T_{n}\left(a^{-1}\right)\right\|_{\infty} \leq\left\|a^{-1}\right\|_{\infty} \leq\left\|a^{-1}\right\|_{W}$ and the latter is uniformly bounded in $n$.

From this lemma, (3.18), (3.7) and (3.21) it follows that

$$
\lim _{n \rightarrow \infty} \exp \left(-C-\operatorname{Tr}\left(T_{n}\left(a^{-1}\right) F_{n}\right)\right) \operatorname{det} B_{n} T_{n}(a b)=1 .
$$

Hence it remains to prove that $\operatorname{Tr} T_{n}\left(a^{-1}\right) F_{n}$ tends to 0 as $n \rightarrow \infty$, which is the most difficult part of the proof. We start with an estimate that follows from a subtle cancellation.

Lemma 3.9. There exists a constant $D$ such that

$$
\sum_{s=-\sqrt{n}}^{\sqrt{n}}\left(a^{-1}\right)_{s}(a)_{N-s} \leq \frac{D}{n^{3 / 4}}
$$

for all $n, N \in \mathbb{N}$ with $N>n$.

Proof. Let $n, N \in \mathbb{N}$ with $N>n$. Define $j^{*}=\sup \left\{j \mid k_{j}<n\right\}$. The proof follows by an induction-like argument with respect to $j^{*}$.

Suppose first that $k_{j^{*}}$ is such that $N-k_{j^{*}}>\sqrt{n} / 2$. In this case split the sum into two parts:

$$
\sum_{s=-\sqrt{n}}^{\sqrt{n}}\left(a^{-1}\right)_{s}(a)_{N-s}=\sum_{|s|<\sqrt{n} / 3}\left(a^{-1}\right)_{s}(a)_{N-s}+\sum_{\sqrt{n} / 3 \leq|s| \leq \sqrt{n}}\left(a^{-1}\right)_{s}(a)_{N-s}
$$


The second sum on the right-hand side of (3.25) is estimated by

$$
\begin{aligned}
& \left|\sum_{\sqrt{n} / 3<|s|<\sqrt{n}}\left(a^{-1}\right)_{s}(a)_{N-s}\right| \\
& \leq\left(\sum_{\sqrt{n} / 3<|s|<\sqrt{n}}\left|\left(a^{-1}\right)_{s}\right|^{2}\right)^{1 / 2}\left(\sum_{\sqrt{n} / 3<|s|<\sqrt{n}}\left|(a)_{N-s}\right|^{2}\right)^{1 / 2} \\
& =\left(\sum_{\sqrt{n} / 3<|s|<\sqrt{n}} \frac{|s|\left|\left(a^{-1}\right)_{s}\right|^{2}}{|s|}\right)^{1 / 2}\left(\sum_{\sqrt{n} / 3<|s|<\sqrt{n}} \frac{|N-s|\left|(a)_{N-s}\right|^{2}}{|N-s|}\right)^{1 / 2} \\
& \leq \frac{\sqrt{3}\left\|a^{-1}\right\|_{B_{2}^{1 / 2}}\|a\|_{B_{2}^{1 / 2}}}{n^{1 / 4} \sqrt{N-\sqrt{n}}} \leq \frac{\sqrt{6}\left\|a^{-1}\right\|_{B_{2}^{1 / 2}}\|a\|_{B_{2}^{1 / 2}}}{n^{3 / 4}},
\end{aligned}
$$

where we used that $N-\sqrt{n} \geq n+1-\sqrt{n}>n / 2$. Note that $\left\|a^{-1}\right\|_{B_{2}^{1 / 2}}$ and $\|a\|_{B_{2}^{1 / 2}}$ are uniformly bounded in $n$ by (2.6).

The first sum on the right-hand side of (3.25) is estimated in a similar way:

$$
\left|\sum_{|s|<\sqrt{n} / 3}\left(a^{-1}\right)_{s}(a)_{N-s}\right| \leq\left\|a^{-1}\right\|_{\mathbb{L}_{2}}\left(\sum_{|s|<\sqrt{n} / 3}\left|(a)_{N-s}\right|^{2}\right)^{1 / 2} .
$$

The term $\left\|a^{-1}\right\|_{\mathbb{L}_{2}}$ is uniformly bounded in $n$. Applying Lemma 3.2, with $t=a$, gives

$$
\begin{aligned}
\sum_{|s|<\sqrt{n} / 3}\left|(a)_{N-s}\right|^{2} & <\sum_{|s|<\sqrt{n} / 3} \frac{\left|\left(F_{a}\left(\mathrm{e}^{F_{a}}-1\right)\right)_{N-s}\right|^{2}}{\left(N-s-k_{j^{*}}\right)(N-s)} \\
& \leq \frac{1}{\left(N-\sqrt{n} / 3-k_{j^{*}}\right)(N-\sqrt{n} / 3)} \sum_{s<\sqrt{n} / 3}\left|\left(F_{a}\left(\mathrm{e}^{F_{a}}-1\right)\right)_{N-s}\right|^{2} \\
& \leq \frac{18}{n^{3 / 2}}\left\|\left(F_{a}\left(\mathrm{e}^{F_{a}}-1\right)\right)\right\|_{\mathbb{L}_{2}} \leq \frac{18}{n^{3 / 2}}\left\|\left(F_{a}\left(\mathrm{e}^{F_{a}}-1\right)\right)\right\|_{W} \\
& \leq \frac{18}{n^{3 / 2}} A_{1}\left(\mathrm{e}^{A_{1}}-1\right) .
\end{aligned}
$$

By combining (3.25), (3.26) and (3.27) we obtain the statement in the case $N-k_{j^{*}}>$ $\sqrt{n} / 2$.

Now suppose $N-k_{j^{*}} \leq \sqrt{n} / 2$. We will then show that the terms that come from $j^{*}$ are negligible. To be precise, define

$$
\begin{aligned}
& c_{1}=\exp \left(\left(\alpha_{j^{*}} z^{k_{j}^{*}}+\alpha_{-j^{*}} z^{-k_{j^{*}}}\right) / \sqrt{k_{j^{*}}}\right), \\
& a_{1}=a c_{1}^{-1} .
\end{aligned}
$$

We will show that

$$
\left|\sum_{s=-\sqrt{n}}^{\sqrt{n}}\left(a^{-1}\right)_{s}(a)_{N-s}-\sum_{s=-\sqrt{n}}^{\sqrt{n}}\left(a_{1}^{-1}\right)_{s}\left(a_{1}\right)_{N-s}\right| \leq\left(\left|\alpha_{j^{*}}\right|+\left|\alpha_{-j^{*}}\right|\right) D_{1} / n,
$$


where $D_{1}$ is a constant independent of $j^{*}, n$ and $N$ that can be expressed in terms of $A_{1}$ and $A_{2}$ only. Redefine $j^{*}$, now with respect to $a_{1}$. If $N-k_{j^{*}}>\sqrt{n} / 2$, then the above arguments show that (3.24) holds for $a_{1}$. By combining this with (3.30) we see that (3.24) also holds for $a$. If however $N-k_{j^{*}}<\sqrt{n} / 2$, then we define $a_{2}$ and $c_{2}$ as in (3.28) and (3.29) and redefine $j^{*}$ with respect to $a_{1}$. We also have that the inequality (3.30) holds with $a_{1}$ replaced by $a_{2}, a$ replaced by $a_{1}$ and $j^{*}$ is with respect to $a_{1}$. If $N-k_{j^{*}}>\sqrt{n} / 2$, then we are again done. Otherwise we continue by defining $a_{3}$ and $c_{3}$ and so on. After a finite number of steps, say $m \leq n+\sqrt{n} / 2-N$, we do find $N-k_{j^{*}}>\sqrt{n} / 2$. At each step $l$ we have the inequality (3.30) with $a$ replaced by $a_{l}$ and $a_{1}$ replaced by $a_{l+1}$ and $j^{*}$ is with respect to $a_{l}$. We can reduce all the inequalities together to the single inequality

$$
\left|\sum_{s=-\sqrt{n}}^{\sqrt{n}}\left(a^{-1}\right)_{s}(a)_{N-s}-\sum_{s=-\sqrt{n}}^{\sqrt{n}}\left(a_{m}^{-1}\right)_{s}\left(a_{m}\right)_{N-s}\right| \leq \frac{D_{1} A_{1}}{n} .
$$

Combining this inequality with the fact that the above arguments show that (3.24) holds for $a_{m}$ leads to the statement.

Hence it remains to prove (3.30). First note that

$$
\left|\sum_{s=-\sqrt{n}}^{\sqrt{n}}\left(a^{-1}\right)_{s}(a)_{N-s}-\sum_{s=-\sqrt{n}}^{\sqrt{n}}\left(a_{1}^{-1}\right)_{s}\left(a_{1}\right)_{N-s}\right| \leq I_{1}+I_{2}+I_{3}
$$

where

$$
\begin{aligned}
I_{1} & =\left|\sum_{s=-\sqrt{n}}^{\sqrt{n}}\left(a^{-1}\right)_{s}(a)_{N-s}-\sum_{s=-\sqrt{n}}^{\sqrt{n}}\left(a_{1}^{-1}\right)_{s}(a)_{N-s}\right| \\
& =\left|\sum_{s=-\sqrt{n}}^{\sqrt{n}}\left(a_{1}^{-1}\left(c_{1}^{-1}-1\right)\right)_{s}(a)_{N-s}\right| \\
I_{2} & =\left|\sum_{s=-\sqrt{n}}^{\sqrt{n}}\left(a_{1}^{-1}\right)_{s}(a)_{N-s}-\sum_{s=-\sqrt{n}}^{\sqrt{n}}\left(a_{1}^{-1}\right)_{s}\left(a_{1}\left(1+\log c_{1}\right)\right)_{N-s}\right| \\
& =\left|\sum_{s=-\sqrt{n}}^{\sqrt{n}}\left(a_{1}^{-1}\right)_{s}\left(a_{1}\left(c_{1}-1-\log c_{1}\right)\right)_{N-s}\right|,
\end{aligned}
$$

and

$$
I_{3}=\left|\sum_{s=-\sqrt{n}}^{\sqrt{n}}\left(a_{1}^{-1}\right)_{s}\left(a_{1} \log c_{1}\right)_{N-s}\right| .
$$

The terms $I_{1}$ and $I_{2}$ can be estimated by the Cauchy-Schwarz inequality,

$$
I_{1} \leq\left\|a_{1}^{-1}\left(c_{1}^{-1}-1\right)\right\|_{\mathbb{L}_{2}}\left(\sum_{s=-\sqrt{n}}^{\sqrt{n}}\left|(a)_{N-s}\right|^{2}\right)^{1 / 2} \leq \frac{\left\|a_{1}^{-1}\right\|_{W}\left\|c_{1}^{-1}-1\right\|_{W}\|a\|_{B_{2}^{1 / 2}}}{\sqrt{N-\sqrt{n}}}
$$

and

$$
I_{2} \leq\left\|a_{1}^{-1}\right\|_{2}\left\|a_{1}\left(c_{1}-1-\log c_{1}\right)\right\|_{2} \leq\left\|a_{1}^{-1}\right\|_{W}\left\|a_{1}\right\|_{W}\left\|c_{1}-1-\log c_{1}\right\|_{W} .
$$


Note that $\left\|a_{1}\right\|_{W},\left\|a_{1}^{-1}\right\|_{W}$ and $\|a\|_{B_{2}^{1 / 2}}$ are all uniformly bounded in $n$ and $N$. Now

$$
\begin{aligned}
&\left\|c_{1}^{-1}-1\right\|_{W} \leq \exp \left(\frac{\left|\alpha_{j^{*}}\right|+\left|\alpha_{-j^{*}}\right|}{\sqrt{k_{j^{*}}}}\right)-1, \\
&\left\|c_{1}-1-\log c_{1}\right\|_{W} \leq \exp \left(\frac{\left|\alpha_{j^{*}}\right|+\left|\alpha_{-j^{*}}\right|}{\sqrt{k_{j^{*}}}}\right)-1-\frac{\left|\alpha_{j^{*}}\right|+\left|\alpha_{-j^{*}}\right|}{\sqrt{k_{j^{*}}}} .
\end{aligned}
$$

Since $k_{j^{*}}>n / 2$ it follows that

$$
I_{1,2} \leq\left(\left|\alpha_{j^{*}}\right|+\left|\alpha_{-j^{*}}\right|\right) D_{2} n^{-1}
$$

for some constant $D_{2}$.

This brings us to the most important part of the proof, namely estimating $I_{3}$. Note that $\log c_{1}=\left(a_{j^{*}} z^{k_{j^{*}}}+\alpha_{-j^{*}} z^{-k^{j^{*}}}\right) / \sqrt{k_{j^{*}}}$. Write

$$
I_{3} \leq I_{31}+I_{32},
$$

where

$$
I_{31}=\left|\frac{\alpha_{k_{j^{*}}}}{\sqrt{k_{j^{*}}}} \sum_{s=-\sqrt{n}}^{\sqrt{n}}\left(a_{1}^{-1}\right)_{s}\left(a_{1}\right)_{N-s-k_{j^{*}}}\right|, I_{32}=\left|\frac{\alpha_{-k_{j^{*}}}}{\sqrt{k_{j^{*}}}} \sum_{s=-\sqrt{n}}^{\sqrt{n}}\left(a_{1}^{-1}\right)_{s}\left(a_{1}\right)_{N-s+k_{j^{*}}}\right| .
$$

The term $I_{32}$ can again be estimated by the Cauchy-Schwarz inequality. The result is that

$$
I_{32} \leq \frac{\sqrt{2}\left|\alpha_{-k_{j^{*}}}\right||| a_{1}^{-1}\left\|_{\mathbb{L}_{2}}\right\| a_{1} \|_{B_{2}^{1 / 2}}}{\sqrt{k_{j^{*}} n}} \leq \frac{2\left|\alpha_{-k_{j^{*}}}\right|\left\|a_{1}^{-1}\right\|_{\mathbb{L}_{2}}\left\|a_{1}\right\|_{B_{2}^{1 / 2}}}{n},
$$

where we used the fact that $N-s+k_{j^{*}} \geq n / 2$ if $|s| \leq \sqrt{n}$ and $k_{j^{*}} \geq n / 2$.

The term $I_{31}$ is more subtle. Since $N>k_{j^{*}}$ we find

$$
\begin{aligned}
\sum_{s=-\sqrt{n}}^{\sqrt{n}}\left(a_{1}^{-1}\right)_{s}\left(a_{1}\right)_{N-s-k_{j^{*}}} & +\sum_{|s|>\sqrt{n}}\left(a_{1}^{-1}\right)_{s}\left(a_{1}\right)_{N-s-k_{j^{*}}}=\sum_{s}\left(a_{1}^{-1}\right)_{s}\left(a_{1}\right)_{N-s-k_{j^{*}}} \\
& =\left(a_{1}^{-1} a_{1}\right)_{N-k_{j^{*}}}=0 .
\end{aligned}
$$

Therefore

$$
I_{31}=\frac{\left|a_{j^{*}}\right|}{\sqrt{k_{j^{*}}}}\left|\sum_{|s|>\sqrt{n}}\left(a_{1}^{-1}\right)_{s}\left(a_{1}\right)_{N-s-k_{j^{*}}}\right| .
$$

Now we estimate the latter expression by the Cauchy-Schwarz inequality again. Note that $\left|N-s-k_{j^{*}}\right|>\sqrt{n} / 2$ if $|s|>\sqrt{n}$. The result is that

$$
I_{31} \leq \frac{2\left|\alpha_{j^{*}}\right||| a_{1}^{-1}\left\|_{B_{2}^{1 / 2}}\right\| a_{1} \|_{B_{2}^{1 / 2}}}{n \sqrt{k_{j^{*}}}} \leq \frac{2^{3 / 2}\left|\alpha_{j^{*}}\right|\left\|a_{1}^{-1}\right\|_{B_{2}^{1 / 2}}\left\|a_{1}\right\|_{B_{2}^{1 / 2}}}{n^{3 / 2}},
$$

where we also used $k_{j^{*}} \geq n / 2$. So from (3.32), (3.33) and (3.34) we find

$$
I_{1,2,3} \leq\left(\left|a_{k_{j^{*}}}\right|+\left|\alpha_{-k_{j^{*}}}\right|\right) D_{3} / n
$$

for some constant $D_{3}$. Now (3.30) follows by (3.31) and (3.35). This proves the statement.

Now we can prove the following corollary by fairly direct estimates. 
Corollary 3.10. We have that

$$
\operatorname{Tr} T_{n}\left(a^{-1}\right) P_{n} H(a) H\left(\widetilde{g^{(2)}}\right) P_{n}=\mathcal{O}\left(n^{-1 / 4}\right),
$$

for $n \rightarrow \infty$.

Proof. A straightforward calculation leads to

$$
\operatorname{Tr} T_{n}\left(a^{-1}\right) P_{n} H(a) H\left(\widetilde{g^{(2)}}\right) P_{n}=\sum_{k_{j}>n} \frac{\alpha_{-j}}{\sqrt{n}} \sum_{s=-n}^{n}\left(a^{-1}\right)_{s}(a)_{k_{j}-s}(n-|s|) .
$$

We estimate each term in the sum with respect to $k_{j}$ separately. So let $k_{j}>n$. Write

$$
\begin{aligned}
\sum_{s=-n}^{n}\left(a^{-1}\right)_{s}(a)_{k_{j}-s}(n-|s|) & =\sum_{s=-\sqrt{n}}^{\sqrt{n}}\left(a^{-1}\right)_{s}(a)_{k_{j}-s}(n-|s|) \\
& +\sum_{\sqrt{n}<|s| \leq n}\left(a^{-1}\right)_{s}(a)_{k_{j}-s}(n-|s|) .
\end{aligned}
$$

After some preparation, the rightmost sum of the right-hand side of (3.38) can be estimated by the Cauchy-Schwarz inequality as before:

$$
\begin{gathered}
\left|\sum_{\sqrt{n}<|s| \leq n}\left(a^{-1}\right)_{s}(a)_{k_{j}-s}(n-|s|)\right| \leq \sum_{\sqrt{n}<|s| \leq n}\left|\left(a^{-1}\right)_{s}(a)_{k_{j}-s}\right||n-s| \\
\quad=\sum_{\sqrt{n}<|s| \leq n} \sqrt{|s| \mid}\left(a^{-1}\right)_{s}\left|\sqrt{k_{j}-s}\right|(a)_{k_{j}-s} \mid \frac{(n-|s|)}{\sqrt{|s|\left(k_{j}-s\right)}} \\
\quad \leq\left\|a^{-1}\right\|_{B_{2}^{1 / 2}}\|a\|_{B_{2}^{1 / 2}} n^{1 / 4},
\end{gathered}
$$

where we used that

$$
\frac{n-|s|}{\sqrt{|s|\left(k_{j}-s\right)}} \leq \sqrt{\frac{n-|s|}{|s|}} \leq n^{1 / 4}
$$

for all $\sqrt{n} \leq|s| \leq n$.

Now consider the left sum on the right-hand side of (3.38):

$$
\begin{aligned}
\left|\sum_{s=-\sqrt{n}}^{\sqrt{n}}\left(a^{-1}\right)_{s}(a)_{k_{j}-s}(n-|s|)\right|=n \mid & \sum_{s=-\sqrt{n}}^{\sqrt{n}}\left(a^{-1}\right)_{s}(a)_{k_{j}-s} \mid \\
& +\left|\sum_{s=-\sqrt{n}}^{\sqrt{n}}\right| s\left|\left(a^{-1}\right)_{s}(a)_{k_{j}-s}\right| .
\end{aligned}
$$

The second sum on the right-hand side of (3.40) can again be estimated by a Cauchy-Schwarz argument, from which it follows that it is of order $n^{-1 / 2}$. The first sum on the right-hand side of (3.40) can be dealt with by using Lemma 3.9 and therefore

$$
\left|\sum_{s=-\sqrt{n}}^{\sqrt{n}}\left(a^{-1}\right)_{s}(a)_{k_{j}-s}(n-|s|)\right|=\mathcal{O}\left(n^{1 / 4}\right)
$$

for $n \rightarrow \infty$. 
Inserting (3.39) and (3.41) into (3.38) and using (3.37) gives

$$
\operatorname{Tr} T_{n}\left(a^{-1}\right) P_{n} H(a) H\left(\widetilde{g^{(2)}}\right) P_{n}=\mathcal{O}\left(n^{-1 / 4}\right) \sum_{k_{j}>n} \alpha_{-j}=\mathcal{O}\left(n^{-1 / 4}\right),
$$

for $n \rightarrow \infty$. This proves the statement.

We are almost at the end of our proof. The final thing we need to show is that the dominant term in $\operatorname{Tr} T_{n}(a) F_{n}$ comes from $\operatorname{Tr} T_{n}\left(a^{-1}\right) P_{n} H(a) H\left(\widetilde{g^{(2)}}\right) P_{n}$, which is small by the previous corollary.

\section{Corollary 3.11.}

$$
\lim _{n \rightarrow \infty} \operatorname{Tr} T_{n}\left(a^{-1}\right) F_{n}=0 .
$$

Proof. Since $W_{n}^{2}=P_{n}$ and by (2.3) we find

$$
\begin{aligned}
\operatorname{Tr} T_{n}\left(a^{-1}\right) F_{n} & =\operatorname{Tr} T_{n}\left(a^{-1}\right) P_{n} H(a) H(\tilde{b}) P_{n}+\operatorname{Tr} T_{n}\left(a^{-1}\right) W_{n} H(\tilde{a}) H(b) W_{n} \\
& =\operatorname{Tr} T_{n}\left(a^{-1}\right) P_{n} H(a) H(\tilde{b}) P_{n}+\operatorname{Tr} W_{n} T_{n}\left(\widetilde{a^{-1}}\right) P_{n} H(\tilde{a}) H(b) W_{n} \\
& =\operatorname{Tr} T_{n}\left(a^{-1}\right) P_{n} H(a) H(\tilde{b}) P_{n}+\operatorname{Tr} T_{n}\left(\widetilde{a^{-1}}\right) P_{n} H(\tilde{a}) H(b) P_{n} .
\end{aligned}
$$

We will only show that $\operatorname{Tr} T_{n}\left(a^{-1}\right) P_{n} H(a) H(\tilde{b}) P_{n} \rightarrow 0$. The right term tends to 0 by the same arguments. Write

$$
\begin{aligned}
T_{n}\left(a^{-1}\right) P_{n} H(a) H(\tilde{b}) P_{n} & =\operatorname{Tr} T_{n}\left(a^{-1}\right) P_{n} H(a) H\left(\tilde{b}-\widetilde{g^{(2)}}-1\right) P_{n} \\
& +\operatorname{Tr} T_{n}\left(a^{-1}\right) P_{n} H(a) H\left(\widetilde{g^{(2)}}\right) P_{n} .
\end{aligned}
$$

Since

$$
\left\|H\left(\tilde{b}-\widetilde{g^{(2)}}-1\right) P_{n}\right\|_{2} \leq \sqrt{n}\left\|b-g^{(2)}-1\right\|_{\mathbb{L}_{2}} \leq \sqrt{n}\left(\mathrm{e}^{\left\|g^{(2)}\right\|_{W}}-\left\|g^{(2)}\right\|_{W}-1\right),
$$

and $\left\|g^{(2)}\right\|_{W} \leq A_{1} / \sqrt{n}$ it follows that

$$
\begin{gathered}
\left|\operatorname{Tr} T_{n}\left(a^{-1}\right) P_{n} H(a) H\left(\widetilde{b}-\widetilde{g^{(2)}}-1\right) P_{n}\right| \leq\left\|T_{n}\left(a^{-1}\right) P_{n} H(a) H\left(\tilde{b}-\widetilde{g^{(2)}}-1\right) P_{n}\right\|_{1} \\
\leq\left\|T_{n}\left(a^{-1}\right)\right\|_{\infty}\left\|P_{n} H(a)\right\|_{2}\left\|H\left(\tilde{b}-\widetilde{g^{(2)}}-1\right) P_{n}\right\|_{2}=\mathcal{O}\left(n^{-\frac{1}{2}}\right),
\end{gathered}
$$

for $n \rightarrow \infty$. By combining this with (3.43) we see that it only remains to estimate $\operatorname{Tr} T_{n}\left(a^{-1}\right) P_{n} H(a) H\left(\widetilde{g^{(2)}}\right) P_{n}$, which was done in Corollary 3.10. This proves the statement.

Now (1.12) follows from Corollary 3.11 and (3.23).

\section{Proof of Theorem 1.1}

We will now show how the condition $\sum\left|\alpha_{j}\right|<\infty$ can be made obsolete when we assume that $\alpha_{-j}=\overline{\alpha_{j}}$. Let $m \in \mathbb{N}$. We split $X_{n}$ into two parts:

$$
X_{n}=X_{n, m}+Y_{n, m}=\sum_{0<|j| \leq m} \frac{\alpha_{j}}{\sqrt{\min \left(\left|k_{j}\right|, n\right)}} \operatorname{Tr} U^{k_{j}}+\sum_{|j|>m} \frac{\alpha_{j}}{\sqrt{\min \left(\left|k_{j}\right|, n\right)}} \operatorname{Tr} U^{k_{j}} .
$$


Since both $X_{n, m}$ and $Y_{n, m}$ are real we find that

$$
\begin{aligned}
\left|\mathbb{E}\left[\mathrm{e}^{\mathrm{i} X_{n}}\right]-\mathbb{E}\left[\mathrm{e}^{\mathrm{i} X_{n, m}}\right]\right| & =\mid \mathbb{E}\left[\mathrm{e}^{\mathrm{i}\left(X_{n, m}+Y_{n, m}\right)}-\mathbb{E}\left[\mathrm{e}^{\mathrm{i} X_{n, m}}\right] \mid \leq \mathbb{E}\left[\left|\mathrm{e}^{\mathrm{i} Y_{n, m}}-1\right|\right]\right. \\
& \leq \mathbb{E}\left[\left|Y_{n, m}\right|\right] \leq \mathbb{E}\left[\left|Y_{n, m}\right|^{2}\right]^{1 / 2}=\left(\sum_{|j|>m}\left|\alpha_{j}\right|^{2}\right)^{1 / 2} .
\end{aligned}
$$

In the last expression we used the fact that the elements $\frac{1}{\sqrt{\min \left(\left|k_{j}\right|, n\right)}} \operatorname{Tr} U^{k_{j}}$ are orthonormal with respect to the Haar measure on $U(n)$. It follows that

$$
\limsup \mid \mathbb{E}\left[\mathrm{e}^{\mathrm{i}\left(X_{n, m}+Y_{n, m}\right)}-\mathbb{E}\left[\mathrm{e}^{\mathrm{i} X_{n, m}}\right] \mid \leq\left(\sum_{|j|>m}\left|\alpha_{j}\right|^{2}\right)^{1 / 2} .\right.
$$

Since $\sum_{|j| \leq m}\left|\alpha_{j}\right|<\infty$, it follows by Theorem 1.2 and (1.5) that

$$
\lim _{n \rightarrow \infty} \mathbb{E}\left[\mathrm{e}^{\mathrm{i} X_{n, m}}\right]=\mathrm{e}^{-\sum_{j=1}^{m}\left|\alpha_{j}\right|^{2}} .
$$

Hence

$$
\begin{aligned}
& \limsup _{n \rightarrow \infty} \mid \mathbb{E}\left[\mathrm{e}^{\mathrm{i}\left(X_{n, m}+Y_{n, m}\right)}-\mathrm{e}^{-\sum_{j=1}^{\infty}\left|\alpha_{j}\right|^{2}}\left|\leq \limsup _{n \rightarrow \infty}\right| \mathbb{E}\left[\mathrm{e}^{\mathrm{i}\left(X_{n, m}+Y_{n, m}\right)}-\mathbb{E}\left[\mathrm{e}^{\mathrm{i} X_{n, m}}\right] \mid\right.\right. \\
& 4.6) \quad+\limsup _{n \rightarrow \infty}\left|\mathbb{E}\left[\mathrm{e}^{\mathrm{i} X_{n, m}}\right]-\mathrm{e}^{-\sum_{j=1}^{m}\left|\alpha_{j}\right|^{2}}\right|+\left|\mathrm{e}^{-\sum_{j=1}^{m}\left|\alpha_{j}\right|^{2}}-\mathrm{e}^{-\sum_{j=1}^{\infty}\left|\alpha_{j}\right|^{2}}\right| \\
& \quad \leq\left(\sum_{|j|>m}\left|\alpha_{j}\right|^{2}\right)^{1 / 2}+\left|\mathrm{e}^{-\sum_{j=1}^{m}\left|\alpha_{j}\right|^{2}}-\mathrm{e}^{-\sum_{j=1}^{\infty}\left|\alpha_{j}\right|^{2}}\right| .
\end{aligned}
$$

If we let $m \rightarrow \infty$ the right-hand side tends to zero.

\section{SOME COMMENTS ON MORE GENERAL $n$-DEPENDENCE}

The $n$-dependence in the symbols we consider is of a special type. Let $U$ be an $n \times n$ unitary matrix randomly chosen with respect to the Haar measure. Consider the random variable $X_{n}$ by

$$
X_{n}(U)=\sum_{|j|>0} \frac{\alpha_{j}(n)}{\sqrt{\min \left(\left|k_{j}(n)\right|, n\right)}} \operatorname{Tr} U^{k_{j}(n)},
$$

where $\alpha_{j}(n)$ now also depends on $n$. Again we assume that for each $n$ we have that $\alpha_{j}(n)=\overline{\alpha_{-j}(n)},\left\{k_{j}(n)\right\}_{j \in \mathbb{N}}$ is a sequence of mutually distinct positive integers and $k_{-j}(n)=-k_{j}(n)$. Define

$$
\sigma_{n}^{2}=2 \sum_{j=1}^{\infty}\left|\alpha_{j}(n)\right|^{2}
$$

and assume that $\sigma_{n} \rightarrow \sigma$ as $n \rightarrow \infty$ for some $\sigma$. A natural question is now under what conditions it is still true that

$$
\lim _{n \rightarrow \infty} \mathbb{E}\left[\mathrm{e}^{\mathrm{i} t X_{n}}\right]=\mathrm{e}^{-t \sigma^{2} / 2},
$$


since then $X_{n}$ converges to a complex normal with mean zero and variance $\sigma^{2}$. Although it is known in some cases that it is true, it will not hold in general.

We will illustrate the subtleties that are involved by an explicit example inspired by [12. Let $f$ be a $C^{\infty}$ function with support within $[-\pi, \pi]$ and let $0<\gamma \leq 1$. Define $k_{j}(n)=j$ and

$$
\alpha_{j}(n)=\frac{\sqrt{\min (|j|, n)}}{2 \pi n^{\gamma}} \hat{f}\left(j / n^{\gamma}\right)
$$

for all $j$ and $n$. Here $\hat{f}$ stands for the Fourier transform of $f$. We assume that

$$
\hat{f}(0)=\int_{\mathbb{R}} f(x) \mathrm{d} x=0 .
$$

The random variable $X_{n}$ can now be rewritten as

$$
X_{n}(U)=\sum_{\mu=1}^{n} f\left(n^{\gamma} \theta_{\mu}\right) .
$$

Since $f$ has compact support, $X_{n}$ only depends on a few eigenvalues, for which $\theta_{\mu}$ is close to zero. If $0<\gamma<1$, then it is true that $X_{n} \rightarrow N\left(0, \sigma^{2}\right)$, where

$$
\sigma^{2}=\frac{1}{4 \pi^{2}} \int|y \| \hat{f}(y)|^{2} \mathrm{~d} y
$$

assuming that the latter is finite. This is proved by Soshnikov [12.

However, the result no longer holds for $\gamma=1$. This case is considered by Hughes and Rudnick in [9] and for the classical compact groups other than $U(n)$ in [10. In these works the authors analyzed the limiting behavior of the moments $\mathbb{E}\left(X_{n}^{m}\right)$ for $m \in \mathbb{N}$ and proved that in general the limiting value of the moments depend on $f$ and are certainly not Gaussian moments. Hence a result such as (5.3) cannot hold. However, if supp $\hat{f} \subset[-2 / m, 2 / m]$, then the $m$-th moment does converge to the $m$-th moment of the normal distribution with mean zero and variance

$$
\sigma^{2}=\frac{1}{4 \pi^{2}} \int_{-\infty}^{\infty} \min (|y|, 1)|\hat{f}(y)|^{2} \mathrm{~d} y .
$$

This phenomenon is called mock-Gaussian behavior in 9 .

\section{ACKNOWLEDGEMENTS}

The presented work was developed while the first author was staying at the Royal Institute of Technology in Stockholm during the spring term of 2006. The authors wish to thank Jens Hoppe for inviting the first author and for his generous hospitality during this period.

The authors also wish to thank Zeev Rudnick for drawing attention to the papers [9] and [10].

\section{REFERENCES}

1. Basor, E., Widom, H., On a Toeplitz determinant identity of Borodin and Okounkov, Integral Equations Operator Theory, 2000, 37(4), 397-401. MR.1780119 (2001g:47042b)

2. Basor, E., Widom, H., Toeplitz and Wiener-Hopf determinants with piecewise continuous symbols, J. Funct. Anal., 1983, 3, 387-413. MR695420 (85d:47026)

3. Borodin, A., Okounkov, A., A Fredholm determinant formula for Toeplitz determinants, Integral Equations Operator Theory, 2000, 37(4), 386-396. MR1780118 (2001g:47042a)

4. Böttcher, A., Silbermann, B., Introduction to large truncated Toeplitz matrices, SpringerVerlag, New York, 1999. MR.1724795 (2001b:47043) 
5. Diaconis, P., Patterns in eigenvalues: The 70th Josiah Willard Gibbs Lecture, Bull. Amer. Math. Soc., 2003, 40(2), 155-178. MR1962294 (2004d:15017)

6. Diaconis, P., Evans, S., Linear functionals of eigenvalues of random matrices, Trans. Amer. Math. Soc., 2001, 353, 2615-2633. MR.1828463 (2002d:60003)

7. Diaconis, P., Shahshahani, M., On the eigenvalues of random matrices, In Studies in Applied Probability. J. Appl. Probab.: Special Vol. 31A, 1994, 49-62. MR.1274717 (95m:60011)

8. Geronimo, J. S., Case, K. M., Scattering theory and polynomials orthogonal on the unit circle, J. Math. Phys., 1979, 20(2), 299-310. MR.519213 (80f:81100)

9. Hughes, C. P., Rudnick, Z., Linear statistics of low-lying zeros of $L$-functions, Quart. J. Math., 2003, 54 (3), 309-333. MR 2013141(2005a:11131)

10. Hughes, C. P., Rudnick, Z., Mock Gaussian behavior for linear statistics of classical compact groups, J. Phys. A, 2003, 36(2), 2919-2932. MR.1986399 (2004e:60012)

11. Rains, E., High powers of random elements of compact Lie groups, Probab. Theory Related Fields, 1997, 107, 219-241. MR1431220 (98b:15026)

12. Soshnikov, A., The central limit theorem for local linear statistics in classical compact groups and related combinatorial identities, Ann. Prob., 2000, 28, 1353-1370. MR1797877 (2002f:15035)

13. Widom, H., Asymptotic behavior of block Toeplitz matrices and determinants, II, Adv. in Math., 1976, 21(1), 1-29. MR0409512 (53:13266b)

14. Wieand, K., Eigenvalue distributions of random unitary matrices, Probab. Theory Related Fields, 2002, 123(2), 202-224. MR.1900322(2003b:60016)

Department of Mathematics, Katholieke Universiteit Leuven, Celestijnenlaan 200 B, 3001 Leuven, Belgium

E-mail address: maurice.duits@wis.kuleuven.be

Current address: Department of Mathematics, California Institute of Technology, 1200 E. California Boulevard, Pasadena, California 91101

Department of Mathematics, Royal Institute of Technology, SE-100 44 Stockholm, SWEDEN

E-mail address: kurtj@kth.se 